\title{
There is so much more to do: Strategies and research needs to support work transitions for persons with chronic mental health conditions
}

\section{Introduction}

Drs. Sumsion and Shaw are pleased to be the coeditors of this special issue of the journal WORK, focusing on mental health and innovative strategies that support work transitions for persons with long term mental health conditions. Dr. Thelma Sumsion is committed to the ongoing application of client-centred practice as it affects clients with severe and persistent mental illness. This goal is clearly connected to enabling their successful entry or return to the workforce. Dr. Lynn Shaw is committed to promoting research on work transitions for persons with disabilities.

Papers in this issue offer a range of interdisciplinary strategies that can promote transitions to productive employment. The authors of these papers include multiple stakeholders; researchers and practitioners from a variety of backgrounds, such as occupational therapists, occupational scientists, vocational rehabilitation professionals, psychiatrists, mental health researchers, nurses, physicians, and educators; employment support agencies; consumer agencies; community agencies; employers and; persons with mental illness/ consumers. What is unique about this issue is the emphasis on the use of cases that offer exemplars of collaborative approaches to support work transitions that include community agency representatives and consumers as coauthors. In addition, this issue of the journal includes diverse types of papers. Case studies include individual narratives (Penhale et al., Cockburn et al.), a collaborative case study (Bergmans et al.), and an organizational case study (Lal and Mercier). Research studies include qualitative investigations of barriers and strategies to support work transitions using document analysis (Krupa et al.), and focus groups (Cowls and Galloway, Moll et al.), and two quantitative studies into mental health outcomes and factors (Jackson et al., and Dewa et al.).

\section{Advancing best practice and research}

To highlight the key contributions the articles make to strategies supporting work transitions, we drew upon Kirsh and colleagues' [1] six principles to guide best practices, promote social change, and advance research on work transitions into mainstream employment for persons with disabilities. These principles suggest that best practices and research must focus on resources and supports across individual, workplace and societal levels.

Principle 1. Establishing a shared view of authentic work participation

This principle, that addresses the desire to contribute to society and to obtain the associated benefits, is expanded by the work of Lal and Mercier, and Moll and colleagues. Both articles underscore efforts and strategies to support a shared view about the capacity of persons with chronic mental illness to participate in productive work occupations. Lal and Mercier provide a case study of an intersectoral approach and the inter organizational challenges in maintaining a shared view and goals for work transitions using authentic 
work places. Moll and colleagues also establish a shared view, through building capacity within the mental health sector to train and hire persons with chronic mental health problems as peer support providers.

Principle 2. Need for reflection on the intervention philosophy supporting work interventions outcomes

Philosophical beliefs in human potential and self actualization are supported by the Cowls and Galloway article that demonstrates how two senior occupational therapists in mental health used observations and reflections of client challenges, and a lack of success in returning to work, to investigate the source of problems with clients and find innovative solutions. Insights from their qualitative study suggest ways to increase client awareness about their illness, and strengths and resources needed to improve career choices, along with developing individual coping strategies for employment success.

Principle 3. Access to supports, professional and workplace

Individuals experiencing mental health challenges need access to relevant supports. Three articles, Sobswale and colleagues, Crane and colleagues, and Cowls and Galloway, emphasize the importance of accessing professional support while transitioning across the multi-staged process of work reentry. All of these articles draw attention to the importance of professional support to clients in assisting them with appraisal and reappraisal of their capacities, and offering opportunities, and a space for feedback through work transitions.

Principle 4. Access to employment and/or accommodations congruent with the needs of the person, the demands of the occupation and resources in the work environment

Case studies by Crane and colleagues and Lal and Mercier as well as the study by Moll et al. underscore this principle by stressing the congruence of matching individual strengths and capacities with appropriate resources and supports in work place environments. Both the Lal and Mercier and Moll et al. articles offer innovative approaches in developing the capacities of organizations to improve their readiness to support work transitions, and in matching persons with chronic mental illness with productive work.

Principle 5. Focus on opportunities to address attitudinal or environment barriers

Krupa and colleagues conducted a qualitative study to help identify the sources and complexity of attitudinal barriers and stigma that limit successful participation in work occupations for persons with chronic mental health conditions. Their study discusses potential strategies that might be considered to eliminate the injustices and consequences of stigma. They also outline strategies for crucial future research.

Principle 6. Applying best practices in and across groups

Ongoing research is required on the work related needs of persons facing mental health challenges. Case studies by Bergman et al., Crane et al. and Sobowale et al. work toward this goal by including narratives and the voices of persons with chronic mental health conditions who have navigated through the challenges of resuming and becoming employed. Insights from these experiences reveal the elements and components of the best practices encountered in placement and supported employment approaches. Ongoing access to supports afforded these consumers with the transformational resources they needed to progress and achieve work potential over time. These case studies should be shared with other consumers and mental health providers to help them reflect together upon the experiences of others, and to identify potential processes or strategies to support best practices in finding productive employment.

Two other articles raise important issues for research into strategies and factors in mental health. Jackson et al. identify the need to expand the realm of secondary outcomes considered in and through work transitions. Secondary health outcomes, such as utilization of emergency, ambulatory care and inpatient services for individuals with a mental illness involved in vocational programs, should be evaluated during the work transition period. Dewa and colleagues focus on a unique sector of employees in education, that of principals. Their research suggests that examining the association of mental health status through self report and workplace conditions can help to identify factors at the macro level within work environments that might be corrected to address and prevent mental health concerns that may lead to work loss.

\section{Summary}

The findings in the articles included in this exciting issue of the journal WORK point to important new areas for research. There is currently a gap in the examination of the socio-cultural and institutional aspects of a work environment, and it is necessary to examine how these factors influence the work transition process for persons with chronic mental health conditions in resuming employment. This issue also highlights the im- 
portance of including the voices of all stakeholders and we hope this emphasis will continue in future research endeavors.

\section{Reference}

[1] B. Kirsh, M. Stergiou-Kita, R. Gewurtz, D. Diredre, T. Krupa and L. Shaw, From Margins to Mainstream: What Do We Know About Work Integration for Persons with Brain Injury, Mental Illness and Intellectual Disability? WORK 32 (2009), pp. 391-405.
Lynn Shaw

Thelma Sumsion

Guest Editors

The University of Western Ontario

School of Occupational Therapy

1201 Western Rd. London Ontario Canada

Tel.: +1 519661211188971

E-mail: leshaw@uwo.ca 